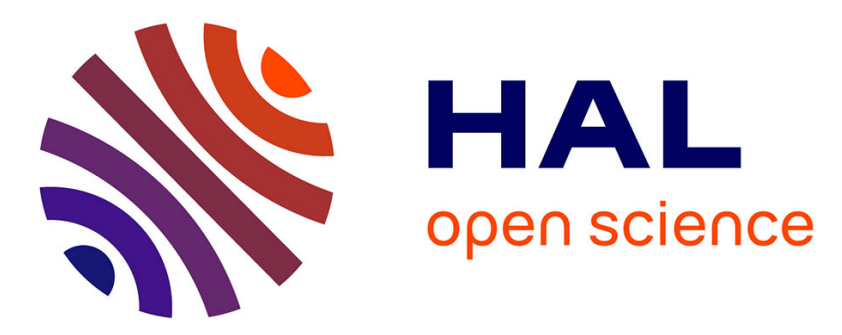

\title{
Chirality distribution in single walled carbon nanotube films by spectroscopic ellipsometry
}

\author{
Y. Battie, D. Jamon, A. En Naciri, J.-S Lauret, A. Loiseau
}

\section{To cite this version:}

Y. Battie, D. Jamon, A. En Naciri, J.-S Lauret, A. Loiseau. Chirality distribution in single walled carbon nanotube films by spectroscopic ellipsometry. Applied Physics Letters, 2013, 102 (9), pp.091909 - 091909-5. 10.1063/1.4795154 . ujm-01025762

\section{HAL Id: ujm-01025762}

\section{https://hal-ujm.archives-ouvertes.fr/ujm-01025762}

Submitted on 11 May 2017

HAL is a multi-disciplinary open access archive for the deposit and dissemination of scientific research documents, whether they are published or not. The documents may come from teaching and research institutions in France or abroad, or from public or private research centers.
L'archive ouverte pluridisciplinaire HAL, est destinée au dépôt et à la diffusion de documents scientifiques de niveau recherche, publiés ou non, émanant des établissements d'enseignement et de recherche français ou étrangers, des laboratoires publics ou privés. 


\section{Chirality distribution in single walled carbon nanotube films by spectroscopic}

ellipsometry

Y. Battie', D. Jamon, A. En Naciri, J.-S. Lauret, and A. Loiseau

Citation: Appl. Phys. Lett. 102, 091909 (2013); doi: 10.1063/1.4795154

View online: http://dx.doi.org/10.1063/1.4795154

View Table of Contents: http://aip.scitation.org/toc/apl/102/9

Published by the American Institute of Physics

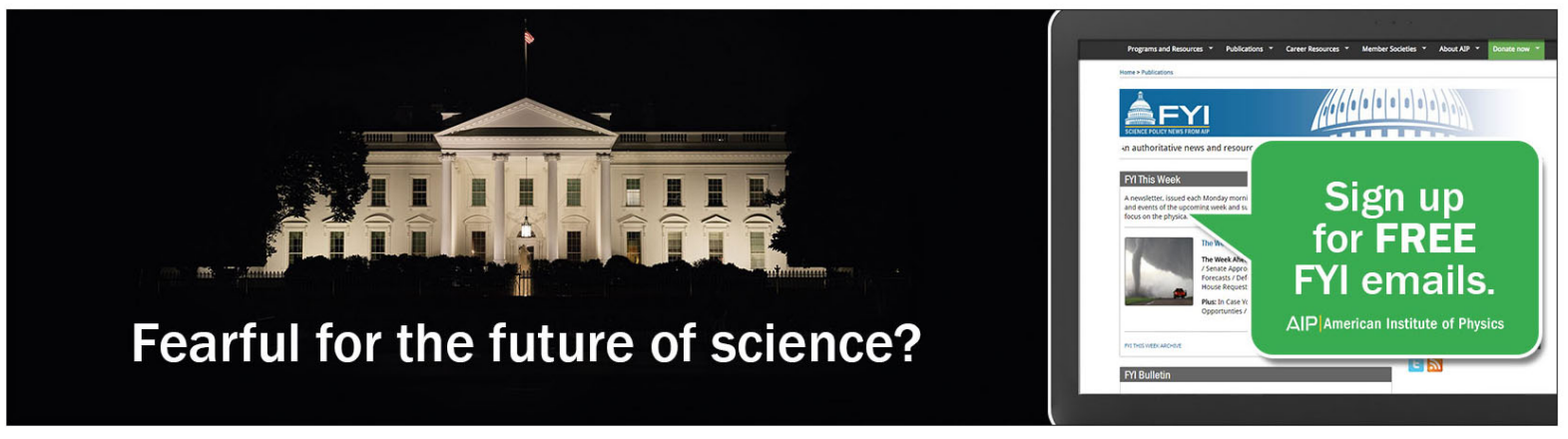




\title{
Chirality distribution in single walled carbon nanotube films by spectroscopic ellipsometry
}

\author{
Y. Battie, ${ }^{1, a)}$ D. Jamon, ${ }^{2}$ A. En Naciri, ${ }^{1}$ J.-S. Lauret, ${ }^{3}$ and A. Loiseau ${ }^{4}$ \\ ${ }^{1}$ LCP-A2MC, Institut Jean Barriol, Université de Lorraine, 1 Bd Arago, 57070 Metz, France \\ ${ }^{2}$ Université de Lyon, Université Jean Monnet, EA 3523, Laboratoire Télécom Claude Chappe, \\ 25 rue du Dr Rémy Annino, 42000 Saint Etienne, France \\ ${ }^{3}$ Laboratoire de Photonique quantique et moléculaire (LPQM), CNRS UMR 8537, ENS Cachan, \\ 94245 Cachan, France \\ ${ }^{4}$ Laboratoire d'étude des microstructures (LEM), ONERA-CNRS UMR 104, 29 Av. de la Division Leclerc, \\ 92322 Chatillon, France
}

(Received 24 January 2013; accepted 27 February 2013; published online 8 March 2013)

\begin{abstract}
We report an experimental technique that determines the chirality distribution in single wall carbon nanotube (SWCNT) films. Films of CoMoCat SWCNTs and SWCNTs enriched in $(6,5)$ chirality are considered. Classical methods like photoluminescence spectroscopy frequently give incomplete distribution. In this way, spectroscopic ellipsometry is used to determine the dielectric function of SWCNT film. The chirality abundance obtained by analysing the ellipsometric data with a tight binding model is compared with that deduced from photoluminescence excitation spectroscopy. We demonstrate that ellipsometry is an efficient tool for a complete and quantitative determination of the chirality distribution and the metallic/semiconducting ratio. (C) 2013 American Institute of Physics. [http://dx.doi.org/10.1063/1.4795154]
\end{abstract}

Single-walled carbon nanotube (SWCNT) is a one dimensional hollow cylindrical nanostructure, which can be viewed as a rolled graphene sheet. A SWCNT is entirely defined by its chiral index $(n, m)$. Depending on their chirality, SWCNTs exhibit various electrical and optical properties and can have a metallic or a semiconducting behaviour. SWCNTs are considered as promising candidates for future optical and electronic devices such as field effect transistor, ${ }^{1}$ gas sensors, ${ }^{2,3}$ optical bolometer, ${ }^{4}$ laser source, ${ }^{5}$ and saturable absorber. ${ }^{6}$ However, as shown previously, ${ }^{3}$ the performances of these devices can be drastically affected by the chirality distribution.

Despite progress towards monochirality samples, ${ }^{7}$ the currently known methods for the fabrication of SWCNTs always produce a mixture of different chiralities. Several post-synthesis separation methods, ${ }^{8-13}$ such as density gradient ultracentrifugation (DGU), ${ }^{12,13}$ have been developed to sort SWCNTs by their electronic properties. The emergence of sorted SWCNTs requires the development of new investigation tools to estimate their chirality distribution. Although the structure of individual SWCNTs can be determined by electronic diffraction ${ }^{14}$ or tunnelling electron microscopy, ${ }^{15}$ both methods are too time consuming to be routinely used to obtain a statistical analysis in a large SWCNT collection.

Due to their one dimensionality, the electronic density of state of SWCNT consists in a series of Van Hove singularity (VHS). The optical properties of semiconducting SWCNT are closely related to the transitions between the first or the second pair of VHS, denoted $S_{11}$ and $S_{22}$, respectively. The optical properties of metallic SWCNTs mainly provide from the transitions between their first VHS pairs, labelled $M_{11}$. As the transition energies $M_{11}, S_{11}$, and $S_{22}$ depend on the nanotube structure, optical spectroscopy, such as absorption

${ }^{a)}$ Electronic mail: yann.battie@univ-lorraine.fr. spectroscopy, ${ }^{16}$ Raman scattering, ${ }^{17}$ or photoluminescence excitation (PLE) spectroscopy, ${ }^{18}$ is a prime tool for the SWCNT characterization. In the case of absorption spectroscopy, overlapping of absorption bands prevents a precise chirality identification. Moreover, the $\pi$ plasmon band in UV region generates an undesirable absorption tail in the visible/ near infrared spectral range, which is frequently unphysically subtracted before the data analysis. ${ }^{16}$ Raman scattering is based on the relationship between the diameter and the frequency of radial breathing modes (RBMs). The signal intensity of the RBM mode strongly depends on the laser excitation wavelength. Indeed, the laser excitation wavelength must be in resonance with the optical transition of SWCNT. Although Raman spectroscopy is highly selective, several laser lines are required to estimate the chirality distribution of a bulk SWCNT sample. Finally, PLE is a powerful characterization technique based on the electronic transitions of SWCNTs. Maximum in the emitted intensity occurred when the excitation and the emission energies are in resonance with optical transitions of SWCNT. ${ }^{18}$ Both energies are specific to SWCNT chirality. However, since metallic carbon nanotubes are not fluorescent, only semiconducting SWCNTs can be detected. The formation of SWCNT bundles also quenches dramatically the fluorescence and prevents the use of PLE for dense film analysis. ${ }^{19,20}$

Spectroscopic ellipsometry ${ }^{21}$ is a non-destructive optical characterisation technique which is highly sensitive to the optical properties of materials. Ellipsometry was recently exploited to characterise thin films with different SWCNT densities. $^{22}$ Using an effective medium approximation, the authors claimed that the interactions between SWCNTs reduce the electronic confinement. Moreover, for high SWCNT density, the film exhibits a metallic behaviour suggesting that the percolation threshold can be estimated by ellipsometry. The optical anisotropy of orientated SWCNT 

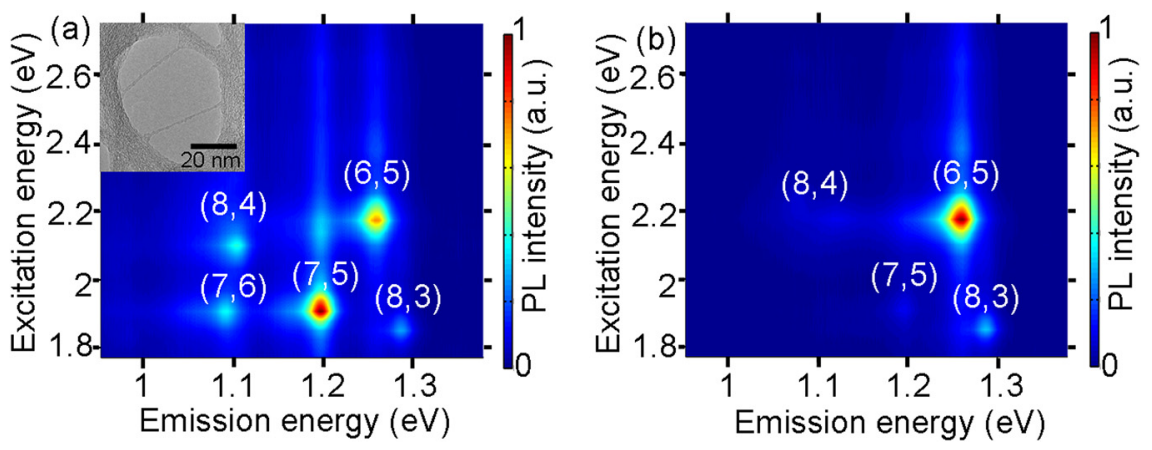

FIG. 1. PLE map of (a) raw and (b) sorted SWCNT solution. In inset, TEM picture of CoMoCat SWCNTs.

films has been also investigated by ellipsometry ${ }^{23,24}$ without taking into account the chirality distribution. This anisotropy was attributed to the dependence between the selection rules and the polarisation. ${ }^{23}$ In the present paper, spectroscopic ellipsometry was used to evaluate both the optical properties and the chirality distribution of raw and DGU enhanced $(6,5)$ chirality SWCNT films. Contrary to previous works, ${ }^{22-24}$ only thick films of randomly orientated SWCNTs are considered. The measured dielectric function of both films was directly correlated to the SWCNT chiralities. By analysing ellipsometric data with a tight binding model, we demonstrate that ellipsometry gives quantitative information on the SWCNT chirality distribution.

Purified CVD growth CoMoCat SWCNTs purchased from SouthWest Nanotechnologies, Inc., are used as raw SWCNTs. Their average diameter, obtained from TEM measurements (inset of Fig. 1), is $0.85 \mathrm{~nm}$. SWCNTs are sorted using non-linear DGU process as described by Gosh et al. ${ }^{13}$ Sorted SWCNT and raw SWCNTs are spread on cleaned glass substrates by filtration process. ${ }^{2,3}$ Both films are composed of randomly orientated SWCNTs network and have a good optical quality. Their thickness, estimated from mechanical profilometry, is around $300 \mathrm{~nm}$. In accordance with transmission spectroscopy measurements (not shown), these films are completely opaque in the considered spectral range (0.8-5 eV).

Figure 1 shows contour maps of the photoluminescence intensity of raw and sorted SWCNT suspensions as a function of excitation and emission wavelengths. The PLE map was obtained on SWCNT suspensions with a home build set up. The excitation wavelength varied from $1.77 \mathrm{eV}$ to $2.75 \mathrm{eV}$. The photoluminescence signal, recorded in the $0.95 \mathrm{eV}$ $1.37 \mathrm{eV}$ spectral range, was corrected by the number of incident photons. In the considered spectral range, 5 hot spots are observed in the PLE maps of raw SWCNT suspension. These come from the resonant $S_{22}$ absorption followed by a radiative recombination through the $S_{11}$ transition. Each hot spot can be associated to a specific chirality by comparing their coordinates with the tabulated ones. ${ }^{18}$ From their relative intensities, the chirality sorting efficiency can be evaluated. The raw SWCNT suspension mainly contains $(7,5),(6,5)$ and $(7,6)$ SWCNTs. Although same $(n, m)$ chiralities are observed in the sorted SWCNTs suspension, the intensities of hot spots are completely modified. The PL intensities of all peaks, excepted to the $(6,5)$ one, are reduced. It suggests a change in the chirality distribution induced by the sorting process and an enrichment in $(6,5)$ SWCNT.

The $\Psi$ and $\Delta$ ellipsometric angles ${ }^{21}$ (not shown) are measured in reflection at a $60^{\circ}$ and $70^{\circ}$ incidence angles on raw SWCNT film and sorted SWCNT film. Considering that both films are sufficiently thick and opaque to neglect the back reflection from the film substrate interface, the films composed of randomly oriented SWCNTs can be modelled as semi-infinite medium. Assuming an isotropic behaviour, the complex effective dielectric function $\varepsilon$ was determined energy by energy from the following equation: ${ }^{21}$

$$
\varepsilon=\sin ^{2} \theta_{0}\left(1+\left(\frac{1-\tan (\Psi) e^{j \Delta}}{1+\tan (\Psi) e^{j \Delta}}\right)^{2} \tan ^{2} \theta_{0}\right)
$$

where $\theta_{0}$ is the angle of incidence.

The dispersion curves extracted from ellipsometric data are shown in Fig. 2. The roots means square error between the complex dielectric functions estimated at $60^{\circ}$ and $70^{\circ}$ angles of incidence (not shown) is around 0.007 for both films confirming their isotropic behaviour. Electronic transitions in perpendicularly polarised SWCNTs have low oscillator strength. ${ }^{24,25}$ In other words, the optical properties of these films are mainly related to SWCNTs polarised along their axis. ${ }^{24,25}$ The imaginary part of the dielectric function of raw SWCNT film is composed of a series of overlapped bands which forms broad structure. Considering the Kataura plot $^{26}$ and the SWCNT average diameter, bands situated at $0.8-1.4 \mathrm{eV}, 1.5-2.5 \mathrm{eV}$, and $2.6-3 \mathrm{eV}$ are attributed to the $\mathrm{S}_{11}$, $\mathrm{S}_{22}$, and $\mathrm{M}_{11}$ transitions, respectively (Fig. 2). The $\mathrm{S}_{33}$ transitions at $3.5 \mathrm{eV}$ are overlapped with the $\pi$ plasmon band. The dielectric function of raw SWCNT film is different to the dielectric function of sorted SWCNT film and is dominated by a wide band centred at $1.22 \mathrm{eV}$. As shown in theoretical works, ${ }^{27}$ each chirality exhibits a specific dielectric function. In agreement with PLE measurements, it suggests that sorted SWCNT film has a narrower chirality distribution. The plasmon band of $\pi$ electrons is also observed at $3.9 \mathrm{eV}$ and 4.1 $\mathrm{eV}$ for raw and sorted SWCNT film, respectively. Its position is highly sensitive to change in the surrounding dielectric environments of SWCNT and gives some insights on the metallic/semiconducting SWCNT ratio. ${ }^{28}$ Thus, the blueshift of the plasmon band of sorted tubes implies a decrease in metallic SWCNT contents. ${ }^{28}$

The effective dielectric function of SWCNT films can be described by a sum of Lorentzian oscillators ${ }^{24}$

$$
\varepsilon(\omega)=\varepsilon_{0}+\sum \frac{f_{(n, m), X j j}}{\omega_{(n, m) ; X j j}^{2}-\omega^{2}+i \gamma_{(n, m) X j j} \omega},
$$

where $\varepsilon_{0}$ is the static dielectric constant and $\omega$ is the photon energy. The parameters $\mathrm{f}_{(\mathrm{n}, \mathrm{m}) \mathrm{Xjj}}, \omega_{(\mathrm{n}, \mathrm{m}) \mathrm{Xjj}}$ and $\gamma_{(\mathrm{n}, \mathrm{m}) \mathrm{Xjj}}$ are, 

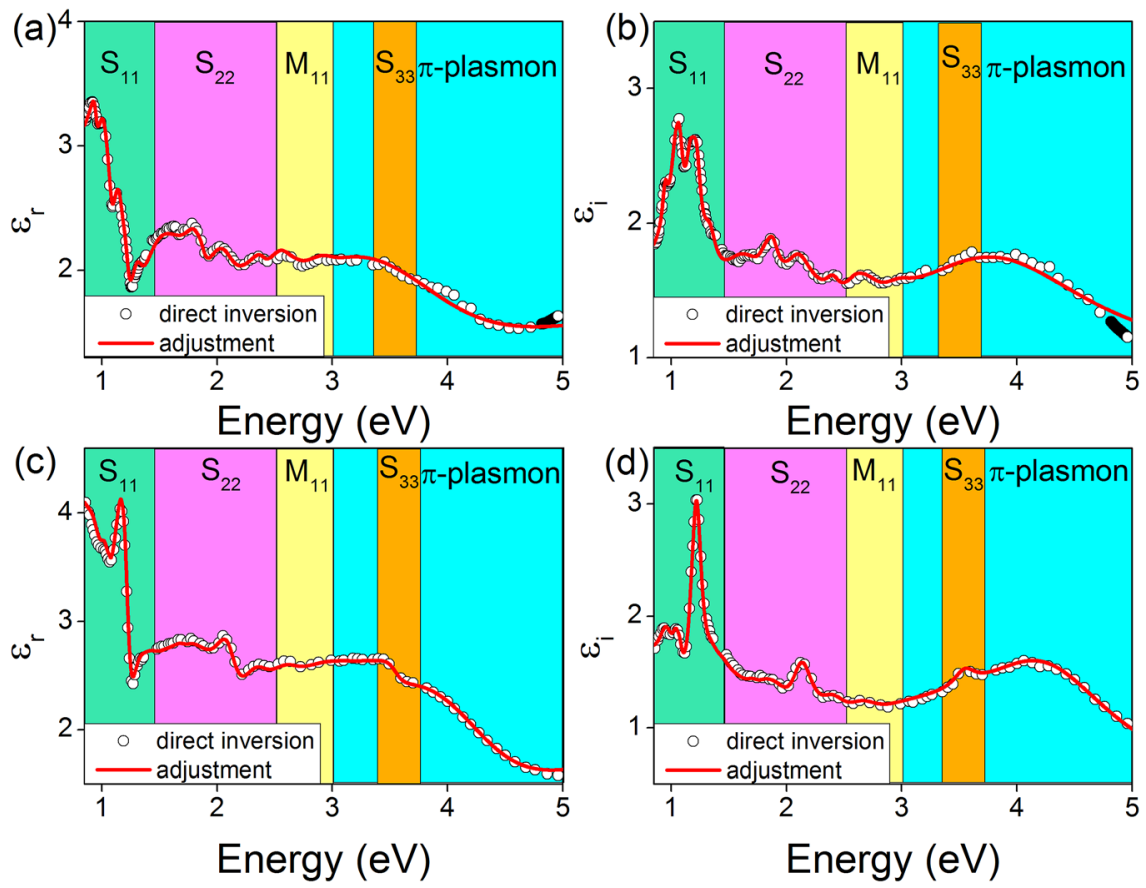

FIG. 2. (a),(c) Real part and (b),(d) imaginary part of dielectric functions of (a),(b) a raw SWCNT film and (c),(d) a sorted SWCNT film, obtained by a direct inversion (dotted curve). The fit results are also reported (solid lines).

respectively, the strength, the resonance energy, and the linewidth of the transition $X_{\mathrm{jj}}$ of a $(n, m)$ SWCNT. Two oscillators per semiconducting SWCNT chirality are used to describe both the $S_{11}$ and $S_{22}$ transitions, while one oscillator per metallic SWCNT chirality is used to model the $\mathrm{M}_{11}$ transition. An other oscillator is added for the $\pi$ plasmon band. This phenomenological model insures the Kramers-Kronig consistency and does not required unphysical background subtraction as absorption spectroscopic measurements. The Levenberg Marquardt algorithm is used to minimize the mean square error (MSE) between the measured and calculated dielectric function. To reduce the number of parameters involved in the ellipsometric model, different numbers of oscillators are tested. The choice of the initial guess model is guided by PLE (Fig. 1) and the previously reported chiralities in CoMoCat sample. ${ }^{7,29}$ Moreover, since ellipsometry estimates both the real part and the imaginary parts of the dielectric function, it gives more information than a simple absorption measurement. Due to the high complexity of the dielectric function, we converge quickly toward a model which consists in 9 semiconducting chirality and 2 metallic chirality.

Fit results are reported in Fig. 2. We found a good agreement between the measured and the calculated dielectric function. The MSE is estimated to 0.02 for both films. All elements of the correlation matrix between each parameter (not shown) are smaller than 0.532 , confirming the independence between the model parameters. The average linewidth of oscillators is $0.16 \mathrm{eV} \pm 0.04 \mathrm{eV}$. This value is larger than the linewidth of isolated SWCNT. ${ }^{30}$ This suggests that SWCNTs are aggregated into bundles. ${ }^{30}$ As reported by Lolli et al., ${ }^{7}$ the $\mathrm{M}_{11}$ transitions comes from the $(6,6)$ and $(7,7)$ metallic SWCNTs. By comparing the $S_{11}$ and $S_{22}$ transition energy to the tabulated one (Fig. 3$),{ }^{18}$ semiconducting SWCNTs are assigned to $(6,5),(7,5),(7,6),(8,3),(8,4)$, $(8,6),(8,7),(9,7)$, and $(9,8)$ SWCNTs. These films contain nearly armchair SWCNTs. ${ }^{7}$ Small variations between the tabulated and the measured transitions energy can be attributed to environmental effects. ${ }^{31}$ Indeed, the dielectric environments are expected to screen the Coulomb interactions and shift the transition energies.

By neglecting the interaction between SWCNTs and the optical response of tubes polarised along their diameter, the oscillator strengths $f_{(n, m), X_{j j}}$ of the transition $X_{j j}$ and the PLE intensity $a_{(n, m)}$ are assumed to be proportional to the concentration $N_{(n, m)}$ in $(\mathrm{n}, \mathrm{m})$ species

$$
\begin{aligned}
f_{(n, m), X_{j j}} & =N_{(n, m)} F_{(n, m), X_{j j}}, \\
a_{(n, m)} & =N_{(n, m)} A_{(n, m)},
\end{aligned}
$$

where $F_{(n, m), X j j}$ and $A_{(n, m)}$ are the intrinsic oscillator strength and PLE intensity of an individual $(\mathrm{n}, \mathrm{m}) \mathrm{SWCNT}$, respectively. $F_{(n, m), X_{j j}}$ and $A_{(n, m)}$ must be evaluated to perform a quantitative estimation of the chirality distribution. The $A_{(n, m)}$ values are extracted from the tabulated ones. ${ }^{32} F_{(n, m), X j j}$ can be expressed as a function of the dipole vectors $\left\langle\Psi_{\mathrm{c}}|\nabla| \Psi_{\mathrm{v}}\right\rangle_{(\mathrm{n}, \mathrm{m}), \mathrm{Xjj}}$ of involved transitions $\mathrm{X}_{\mathrm{jj}}{ }^{32}$

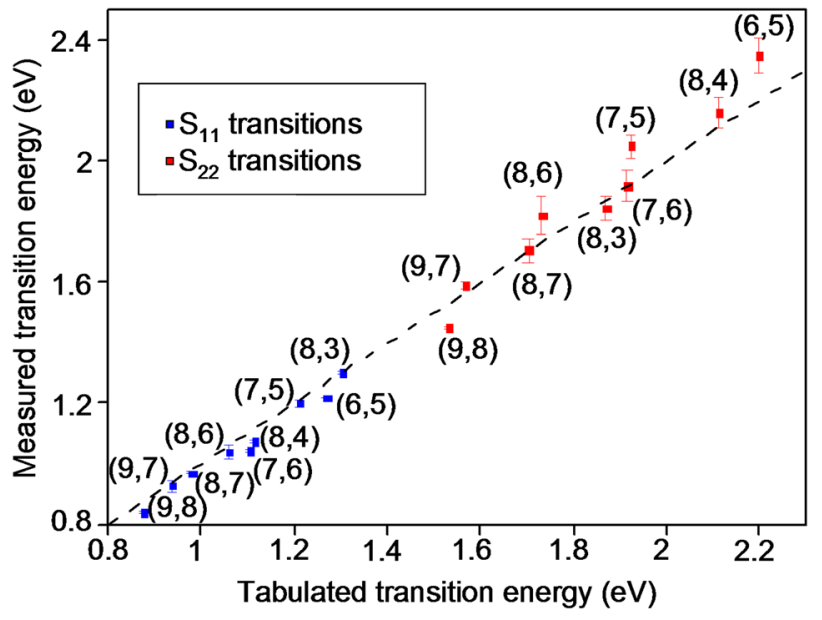

FIG. 3. Comparison between the tabulated and measured $S_{11}$ (blue dots) and $\mathrm{S}_{22}$ (red dots) transition energies. 


$$
F_{(n, m), X_{j j}} \propto \frac{\left\langle\Psi_{c}|\nabla| \Psi_{v}\right\rangle_{(n, m), X_{j j}}^{2}}{\omega_{j j}^{2}}
$$

where $\Psi_{\mathrm{c}}$ and $\Psi_{\mathrm{v}}$ are the wave functions of the conduction and valence bands, respectively. The dipole vector of a SWCNT polarized along its axis can be expressed by using tight binding theory. ${ }^{33}$ The model is based on the relationship

$$
\begin{gathered}
\left\langle\Psi_{c}|\nabla| \Psi_{v}\right\rangle_{(n, m), X j j} \propto \frac{\mu_{1} \sin \left(\frac{\pi}{6}-\theta\right)+\mu_{2} \cos \left(\frac{\pi}{6}-\theta\right)}{\omega_{X j j}}, \\
\mu_{1}=\cos \left(k_{y} a\right)-\cos \left(\frac{k_{y} a}{2}\right) \cos \left(\frac{\sqrt{3} k_{x} a}{2}\right) \\
\mu_{2}=\sqrt{3} \sin \left(\frac{k_{y} a}{2}\right) \sin \left(\frac{\sqrt{3} k_{x} a}{2}\right)
\end{gathered}
$$

where $\theta$ is the chiral angle, $a=0.246 \mathrm{~nm}$ is the atomic lattice constant, and $\left(k_{x}, k_{y}\right)$ are the coordinates of the wave vector $\mathbf{k}$. More complex model, beyond the aims of this paper, can be used to take into account exitonic effect or trigonal wrapping.

Zone folding approximation allows explaining the wave vector as a function of the reciprocal lattice vectors $\mathbf{K}_{\mathbf{1}}$ and $\mathbf{K}_{\mathbf{2}}$ of the 2 D Brillouin zone ${ }^{33}$

$$
\mathbf{k}=\mu \mathbf{K}_{\mathbf{1}}+\frac{k}{\left|\mathbf{K}_{\mathbf{2}}\right|} \mathbf{K}_{\mathbf{2}} \quad\left(1 \leq \mu \leq N_{h},-\frac{\pi}{|\mathbf{T}|}<k<\frac{\pi}{|\mathbf{T}|}\right)
$$

where $\mathbf{T}$ is the translational vector along the tube axis and $N_{h}$ is the number of hexagons in the SWCNT unit cell.

Fig. 4 summarizes the diameter dependence of the intrinsic oscillator strength of a $(n, m)$ tubes normalised to the oscillator strength of the $S_{11}$ transition of $(6,5)$ SWCNT. Consistent with recent theoretical works, ${ }^{32,33}$ the oscillator strength increases linearly with the tube diameter. This justifies the necessity to include the intrinsic oscillator strength in the data analysis. Moreover, the intrinsic oscillator strength of the $S_{11}$ transition band is higher than the $S_{22}$ one. As a consequence, the $S_{11}$ bands found by ellipsometry are more pronounced than the $S_{22}$ bands. Thus, the fitted parameters

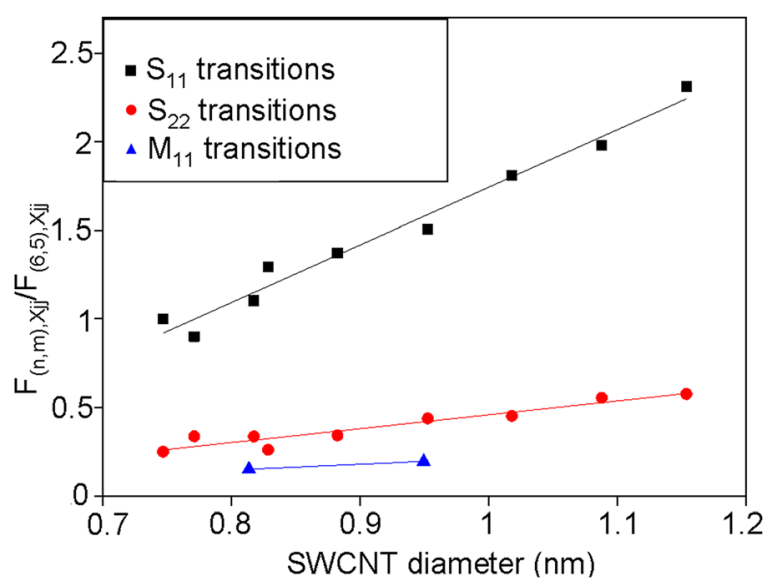

FIG. 4. Diameter dependence of the $\mathrm{S}_{11}, \mathrm{~S}_{22}$, and $\mathrm{M}_{11}$ oscillator strengths.

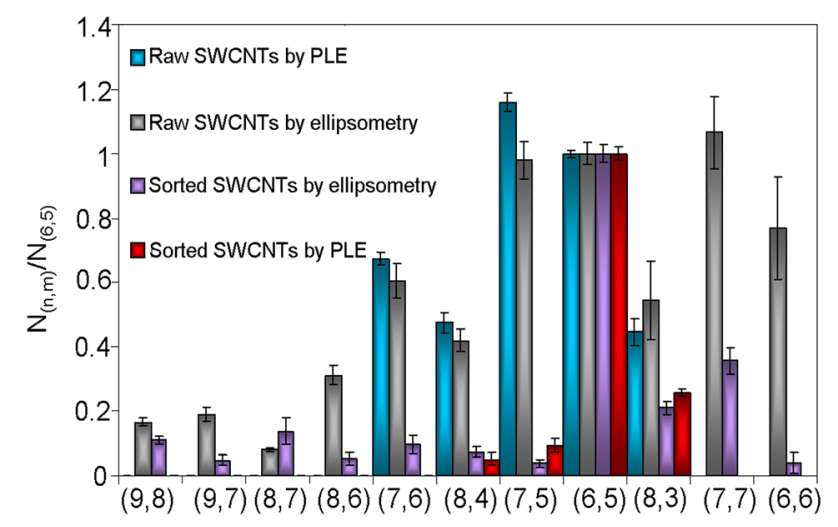

FIG. 5. Chirality distributions of raw SWCNTs and sorted SWCNTs obtained by PLE and ellipsometry.

of $S_{11}$ oscillators are determined with a better accuracy than the $S_{22}$ ones (not shown). In the following, only the oscillator strengths of the $S_{11}$ transitions of semiconducting SWCNTs are considered to predict the semiconducting SWCNT distribution. Note that the oscillator strengths of the $\mathrm{M}_{11}$ transitions are also used to determine the amount of metallic SWCNT.

Fig. 5 compares the abundance of each SWCNT species in both films deduced from ellipsometry and PLE. This was calculated by dividing the oscillator strengths found by ellipsometry and the PLE intensities by their intrinsic values. Both methods give similar chirality distributions. Since quantification of semiconducting tubes from PLE is a well established technique, ${ }^{29}$ this demonstrates the feasibility of ellipsometry for the estimation of the chirality distribution. Metallic and large diameter semiconducting SWCNTs such as $(6,6),(7,7),(9,8),(9,7),(8,7)$, and $(8,6)$ SWCNTs are not detected by PLE. Indeed metallic SWCNTs are not fluorescent. The $S_{11}$ and $S_{22}$ transition energies of larger semiconducting SWCNTs are too low to coincide with the accessible experimental spectral range of PLE measurements. On the contrary, ellipsometry gives insights on the whole population of SWCNTs. Raw SWCNTs contains $16 \% \pm 2 \%$ of $(6,5)$ SWCNT and $70 \% \pm 2.4 \%$ of semiconducting carbon nanotubes. The $(6,5)$ concentration is 3 times higher in the sorted film revealing efficiency of the sorting process. In accordance to the blue shift of the $\pi$ plasmon band, the concentration of semiconducting SWCNT in the sorted film has increased to $82 \% \pm 2.5 \%$. However, a large number of metallic SWCNTs still remained. Iterative orthogonal sorting technique, as suggested by Green et al., ${ }^{34}$ can be used to improve the chirality enrichment by removing metallic SWCNTs in a second DGU step. This argues in favour of ellipsometry to get a complete and quantitative chirality distribution.

In summary, we have highlighted the complex dielectric function of CoMoCat and (6,5) enriched SWCNTs films. We have demonstrated that ellipsometry provides a robust and straightforward tool for tailoring the $(n, m)$ species in macroscopic SWCNT ensembles. Coupled, with tight binding model, ellipsometry measurement gives a complete and quantitative chirality distribution. It was particularly useful to control the overall success of a chirality or semiconducting SWCNT enrichment. 
${ }^{1}$ M. Steiner, M. Engel, Y.-M. Lin, Y. Wu, K. Jenkins, D. B. Farmer, J. J. Humes, N. L. Yoder, J.-W. T. Seo, A. A. Green, M. C. Hersam, R. Krupke, and P. Avouris, Appl. Phys. Lett. 101, 053123 (2012).

${ }^{2}$ Y. Battie, O. Ducloux, P. Thobois, N. Dorval, J. S. Lauret, B. Attal-Trétout, and A. Loiseau, Carbon 49, 3544-3552 (2011).

${ }^{3}$ Y. Battie, L. Gorintin, O. Ducloux, P. Thobois, P. Bondavalli, G. Feugnet and A. Loiseau, Analyst 137, 2151-2157 (2012).

${ }^{4}$ M. E. Itkis, F. Borondics, A. Yu, and R. C. Haddon, Science 312, 413-416 (2006).

${ }^{5}$ E. Gaufrès, N. Izard, X. Le Roux, D. Marris-Morini, S. Kazaoui, E. Cassan, and L. Vivien, Appl. Phys. Lett. 96, 231105 (2010).

${ }^{6}$ H. Nong, M. Gicquel, L. Bramerie, M. Perrin, F. Grillot, C. Levallois, A. Maalouf, and S. Loualiche, Appl. Phys. Lett. 96, 061109 (2010).

${ }^{7}$ G. Lolli, L. Zhang, L. Balzano, N. Sakulchaicharoen, Y. Tan, and D. E. Resasco, J. Phys. Chem. B 110, 2108-2115 (2006).

${ }^{8}$ R. Krupke, S. Linden, M. Rapp, and F. Hennrich, Adv. Mater. 18, 1468-1470 (2006).

${ }^{9}$ C. Ménard-Moyon, N. Izard, E. Doris, and C. Mioskowski, J. Am. Chem. Soc. 128, 6552-6553 (2006).

${ }^{10}$ P. C. Collins, M. S. Arnold, and P. Avouris, Science 292, 706-709 (2001).

${ }^{11}$ M. Zheng and E. D. Semke, J. Am. Chem. Soc. 129, 6084-6085 (2007).

${ }^{12}$ M. S. Arnold, A. A. Green, J. F. Hulvat, S. I. Stupp, and M. C. Hersam, Nat. Nanotechnol. 1, 60-65 (2006).

${ }^{13}$ S. Ghosh, S. M. Bachilo, and R. B. Weisman, Nat. Nanotechnol. 5, 443-450 (2010).

${ }^{14}$ L-C. Qin, Phys. Chem. Chem. Phys. 9, 31-48 (2007).

${ }^{15}$ J. Wildoer, L. Venema, A. Rinzler, R. Smalley, and C. Dekker, Nature 391, 59-62 (1998).

${ }^{16}$ N. Nair, M. L. Usrey, W.-J. Kim, R. D. Braatz, and M. S. Strano, Anal. Chem. 78, 7689-7696 (2006).

${ }^{17}$ A. Jorio, A. P. Santos, H. B. Ribeiro, C. Fantini, M. Souza, J. P. M. Vieira, C. A. Furtado, J. Jiang, R. Saito, L. Balzano, D. E. Resasco, and M. A. Pimenta, Phys. Rev. B 72, 075207 (2005).

${ }^{18}$ S. M. Bachilo, M. S. Strano, C. Kittrell, R. H. Hauge, R. E. Smalley, and R. B. Weisman, Science 298, 2361-2366 (2002).
${ }^{19}$ T. J. McDonald, C. Engtrakul, M. Jones, G. Rumbles, and M. J. Heben, J. Phys. Chem. B 110, 25339-25346 (2006).

${ }^{20}$ J.-S. Lauret, C. Voisin, G. Cassabois, C. Delalande, P. Roussignol, O. Jost, and L. Capes, Phys. Rev. Lett. 90, 057404 (2003).

${ }^{21}$ R. M. A. Azzam and N. M. Bashara, Ellipsometry and Polarized Light (North-Holland Personal Library, 1987).

${ }^{22}$ G. Fanchini, H. E. Unalan, and M. Chhowalla, Appl. Phys. Lett. 88, 191919 (2006).

${ }^{23}$ Y. Battie, D. Jamon, J.-S. Lauret, A. En Naciri, L. Broch, and A. Loiseau, Carbon 50, 4673-4679 (2012).

${ }^{24}$ G. Fanchini, S. Miller, B. B. Parekh, and M. Chhowalla, Nano Lett. 8, 2176-2179 (2008).

${ }^{25}$ H. Ajiki and T. Ando, Physica B 201, 349-352 (1994).

${ }^{26}$ H. Kataura, Y. Kumazawa, Y. Maniwa, I. Umezu, S. Suzuki, Y. Ohtsuka, and Y. Achiba, Synth. Met. 103, 2555-2558 (1999).

${ }^{27}$ G. Y. Guo, K. C. Chu, D.-S. Wang, and C.-G. Duan, Phys. Rev. B 69, 205416 (2004).

${ }^{28}$ Y. R. Park, W.-J. Kim, M. J. Ko, N. K. Min, and C. J. Lee, Nanoscale 4, 6532-6536 (2012).

${ }^{29}$ S. M. Bachilo, L. Balzano, J. E. Herrera, F. Pompeo, D. E. Resasco, and R. B. Weisman, J. Am. Chem. Soc. 125, 11186-11187 (2003).

${ }^{30}$ M. J. O'Connell, S. M. Bachilo, C. B. Huffman,V. C. Moore, M. S. Strano, E. H. Haroz, K. L. Rialon, P. J. Boul, W H. Noon, C. Kittrell, J. Ma, R. H. Hauge, R. B. Weisman, and R. E. Smalley, Science 297, 593-596 (2002).

${ }^{31}$ O. A. Dyatlova, J. Gomis-Bresco, E. Malic, H. Telg, J. Maultzsch, G. Zhong, J. Geng, and U. Woggon, Phys. Rev. B 85, 245449 (2012).

${ }^{32}$ Y. Oyama, R. Saito, K. Sato, J. Jiang, G. G. Samsonidze, A. Gruneis, Y. Miyauchi, S. Maruyama, A. Jorio, G. Dresselhaus, and M. S. Dresselhaus, Carbon 44, 873-879 (2006).

${ }^{33}$ J. Jiang, R. Saito, A. Gruneis, G. Dresselhaus, and M. S. Dresselhaus, Carbon 42, 3169-3176 (2004).

${ }^{34}$ A. A. Green and M C. Hersam, Adv. Mater. 23, 2185-2190 (2011). 